\title{
Lactobacillus reuteri FYNLJ109L1 Attenuating Metabolic Syndrome in Mice via Gut Microbiota Modulation and Alleviating Inflammation
}

\author{
Bo Yang ${ }^{1,2,3} \mathbb{D}$, Fuli Zheng ${ }^{1,2}$, Catherine Stanton ${ }^{3,4,5} \mathbb{D}$, Reynolds Paul Ross ${ }^{3,4} \mathbb{D}$, Jianxin Zhao ${ }^{1,2,6}$, \\ Hao Zhang $1,2,6,7, * \mathbb{D}$ and Wei Chen $1,2,6$
}

1 State Key Laboratory of Food Science and Technology, Jiangnan University, Wuxi 214122, China; bo.yang@jiangnan.edu.cn (B.Y.); zhengfuli66@163.com (F.Z.); zhaojianxin@jiangnan.edu.cn (J.Z.); chenwei66@jiangnan.edu.cn (W.C.)

2 School of Food Science and Technology, Jiangnan University, Wuxi 214122, China

3 International Joint Research Laboratory for Pharmabiotics \& Antibiotic Resistance, Jiangnan University, Wuxi 214122, China; catherine.stanton@teagasc.ie (C.S.); p.ross@ucc.ie (R.P.R.)

4 APC Microbiome Ireland, University College Cork, T12 K8AF Cork, Ireland

5 Teagasc Food Research Centre, Moorepark, Fermoy, P61 C996 Co. Cork, Ireland

6 National Engineering Research Center for Functional Food, Jiangnan University, Wuxi 214122, China

7 Wuxi Translational Medicine Research Center, Jiangsu Translational Medicine Research Institute Wuxi Branch, Wuxi 214122, China

* Correspondence: zhanghao61@jiangnan.edu.cn; Tel./Fax: +86-0510-8591-2155

check for

updates

Citation: Yang, B.; Zheng, F.; Stanton, C.; Ross, R.P.; Zhao, J.; Zhang, H.;

Chen, W. Lactobacillus reuteri

FYNLJ109L1 Attenuating Metabolic

Syndrome in Mice via Gut Microbiota Modulation and Alleviating

Inflammation. Foods 2021, 10, 2081.

https://doi.org/10.3390/foods10092081

Academic Editor: Mary

Anne Amalaradjou

Received: 29 June 2021

Accepted: 31 August 2021

Published: 2 September 2021

Publisher's Note: MDPI stays neutral with regard to jurisdictional claims in published maps and institutional affiliations.

Copyright: (c) 2021 by the authors. Licensee MDPI, Basel, Switzerland. This article is an open access article distributed under the terms and conditions of the Creative Commons Attribution (CC BY) license (https:/ / creativecommons.org/licenses/by/ $4.0 /)$.
Abstract: Metabolic syndrome is caused by an excessive energy intake in a long-term, high-fat and/or high-sugar diet, resulting in obesity and a series of related complications, which has become a global health concern. Probiotics intervention can regulate the gut microbiota and relieve the systemic and chronic low-grade inflammation, which is an alternative to relieving metabolic syndrome. The aim of this work was to explore the alleviation of two different Lactobacillus reuteri strains on metabolic syndrome. Between the two L. reuteri strains, FYNLJ109L1 had a better improvement effect on blood glucose, blood lipid, liver tissue damage and other related indexes than NCIMB 30242. In particular, FYNLJ109L1 reduced weight gain, food intake and fat accumulation. Additionally, it can regulate the gut microbiota, increase IL-10, and reduce IL- 6 and tumor necrosis factor- $\alpha$ (TNF- $\alpha$ ), as well as liver injury, and further reduce insulin resistance and regulate lipid metabolism disorders. In addition, it could modulate the gut microbiota, particularly a decreased Romboutsia and Clostridium sensu stricto-1, and an increased Acetatifactor. The results indicated that FYNLJ109L1 could improve metabolic syndrome significantly via alleviating inflammation and gut microbiota modulation.

Keywords: Lactobacillus reuteri; metabolic syndrome; gut microbiota

\section{Introduction}

Metabolic syndrome (MS) is a clinical syndrome caused by obesity, impaired glucose regulation, hypertension, dyslipidemia and insulin resistance, leading to the occurrence and development of cardiovascular disease (CVD), hypertension, type 2 diabetes (T2D), and non-alcoholic fatty liver disease (NAFLD) [1], which has become a catastrophic epidemic globally [2]. MS prevalence is currently increasing in both developed and developing countries, with a statistically significant prevalence of $20-25 \%$ of MS in adult buckles worldwide [3]. Due to additional evidence, it is widely believed that dietary changes resulting in an excess nutrition could lead to MS. Different dietary structures could affect the weight difference and metabolism regulation directly, and can change the gut microbiota [4], for instance, mice fed with a high-fat diet (HFD) for a long time showed an imbalanced gut microbiota [5]. The decrease in health-promoting bacteria such as lactic acid bacteria and butyric acid producers and the increase in harmful bacteria such as pro-inflammatory and 
pathogenic bacteria are important reasons for the occurrence of chronic inflammation and metabolism disorders [6,7].

The main treatments for MS are lifestyle change and medication. However, lifestyle changes need to be maintained for a long time, and drug treatment, although effectively, may have undesirable side-effects, particularly liver damage [8]. Therefore, there is an urgent need for a safe and effective treatment that can alleviate MS. Notably, probiotics, which are considered safe and basically have no side effects, are considered to have a good intervention effect on a variety of diseases including MS [9]. Lactobacillus is generally recognized as safe and is one of the widely used probiotics for mammals [10]. It demonstrates a key role in regulating the host metabolism, and the active metabolites produced through metabolism have positive effects on the gut microbiota [11,12]. For example, L. reuteri, as a native resident of the human and animal gastrointestinal tracts, has been verified in animal experiments to reduce insulin resistance, the formation of hepatic steatosis, hyperlipidemia, along with the prevention of elevated blood sugar and related MS [13,14]. It was reported that different strains, even from the same species, had different effects on metabolic disorders. Mice intervened with $L$. reuteri ATCC PTA 4659 showed a significantly lower body weight than that in the control group, which was significantly increased in the L. reuteri L6798-treated group [15]. However, the relationship between the host gut microbiota, chronic inflammation, and metabolic disorders has not been adequately investigated with different $L$. reuteri intervention strategies. $L$. reuteri NCIMB 30,242 with a high BSH activity can affect the serum cholesterol in vitro, in rats, and in clinical trials [14,16], which is related to cardiovascular health; however, we presume that it might have potential benefits on metabolic disorders. In the current work, two L. reuteri strains FYNLJ109L1 and NCIMB 30,242 with the same origins, isolated from a piglet fecal sample, were used to investigate the remission effect of different $L$. reuteri strains on the MS induced by an HFD and were compared to explore the correlation between the gut microbiota, inflammation, and MS.

\section{Materials and Methods}

\subsection{Bacterial Preparation}

Two L. reuteri strains were used in this study, in which FYNLJ109L1, originally isolated from pig fecal samples in Lijiang, Yunnan Province, China, was deposited at the Collection Center of Food Microbiology (CCFM)-Jiangnan University, and L. reuteri NCIMB 30,242 was from a porcine origin and with cholesterol lowering capabilities [16,17]. The bacteria were sub-cultured three times in an MRS broth. Then the bacteria were collected via centrifugation at $6000 \times g$ for $15 \mathrm{~min}$. The cell pellets were mixed with a cryoprotectant, which contained $13 \%$ skim milk, $2 \%$ trehalose and $2 \%$ sucrose, and lyophilized and stored at $4{ }^{\circ} \mathrm{C}$.

\subsection{Animals Experiment Design}

The animal experiment was approved by the Animal Ethics Committee of Jiangnan University (JN. No20200630c1201130(121)), and the whole experimental process conformed to the Jiangsu Provincial Measures for the Control of Experimental Animals. Forty-eight C57BL/6J mice (5-week-old, male) without specific pathogens were obtained from the Institute of Model Zoology, Nanjing University (Nanjing, Jiangsu, China). Mice were raised in a specific pathogen-free (SPF) facility under standard conditions (constant temperature of $20 \pm 2{ }^{\circ} \mathrm{C}$, humidity of $50 \pm 5 \%$, and 12-hour light-dark cycle).

Mice were randomly divided into six groups (each $n=8$ ), including one control group, one HFD group, two drug control groups (Simvastatin, Metformin) and two L. reuteri intervention groups (Table 1). The control mice were fed with a normal control diet (NC, total energy $3.6 \mathrm{kcal} / \mathrm{g}, 10 \%$ fat), and the other mice were fed with an HFD (total energy $5.0 \mathrm{kcal} / \mathrm{g}, 60 \% \mathrm{fat})$. During the period of diet induction, the two L. reuteri groups were given $0.2 \mathrm{~mL}$ of freeze-dried bacterial powder resuspended with physiological saline $\left(10^{9} \mathrm{CFU}\right)$, and the drug groups were administrated with simvastatin at $3 \mathrm{mg} /(\mathrm{kg} \mathrm{BW})$ and metformin at $150 \mathrm{mg} /(\mathrm{kg} \mathrm{BW})$, respectively. After one week of acclimatization, all 
the mice were gavaged 6 times each week, and their body weight and food intake were monitored weekly. The whole trial lasted for 14 weeks. An oral glucose tolerance test (OGTT) was carried out at the 13th week of the trial. The body function of the mice was basically restored, and fecal samples were obtained in 14th week. Then, the mice were sacrificed for blood and tissue collection. The liver tissues were immersed in $4 \%$ neutral paraformaldehyde solution for fixation, and stool samples and the rest of the tissue samples were frozen at $-80^{\circ} \mathrm{C}$.

Table 1. Treatment of each group.

\begin{tabular}{ccc}
\hline Group & Diet & Gavage \\
NC & NC & $0.2 \mathrm{~mL}$ physiological saline + lyophilized protection agents \\
HFD & HFD & $0.2 \mathrm{~mL}$ physiological saline + lyophilized protection agents \\
Simvastatin & HFD & $0.2 \mathrm{~mL}$ physiological saline $+3 \mathrm{mg} / \mathrm{kg} / \mathrm{BW}$ simvastatin + lyophilized protection agents \\
Metformin & HFD & $0.2 \mathrm{~mL}$ physiological saline $+150 \mathrm{mg} / \mathrm{kg} / \mathrm{BW}$ metformin + lyophilized protection agents \\
FYNLJ109L1 & HFD & $0.2 \mathrm{~mL}$ physiological saline $+5 \times 10^{9} \mathrm{CFU} / \mathrm{mL}$ FYNLJ109L1 + lyophilized protection agents \\
NCIMB 30242 & HFD & $0.2 \mathrm{~mL}$ physiological saline $+5 \times 10^{9} \mathrm{CFU} / \mathrm{mL}$ NCIMB 30,242 + lyophilized protection agents \\
\hline
\end{tabular}

\subsection{Serum Biochemical Analysis of Blood Lipid Contents}

After the blood was left standing for $3 \mathrm{~h}$, the serum was collected by centrifuging $(1000 \times g, 15 \mathrm{~min})$. Serum lipids were measured by automatic biochemical analyzer (SELECRTA-E, Vital Scientific, Van Selavig, Netherlands), including triacylglycerol (TG), total cholesterol (TC), low-density lipoprotein cholesterol (LDL-C) and high-density lipoprotein cholesterol (HDL-C). In addition, the ratio of LDL-C to HDL-C was calculated as the atherosclerosis index (AI).

\subsection{Glucose and Insulin Tests}

Fasting blood glucose at $0 \mathrm{~h}$ was measured by glucose meter (Roche, Mannheim, Germany) after the mice were fasted overnight. Then, $2 \mathrm{~g} /(\mathrm{kg} \mathrm{BW})$ of glucose was administered to the mice, and the concentrations of blood glucose were measured 30,60 and $120 \mathrm{~min}$ later, respectively. According to the calculation time and the area under the curve of blood glucose value (AUCglucose), a glucose tolerance value was obtained. The fasting serum insulin content in the mice was determined by a commercialized kit (Nanjing Senbeijia Biological Technology, Nanjing, Jiangsu, China). The homeostasis model assessment of insulin resistance was used to evaluate the insulin resistance, and the calculation was performed as follow: fasting blood glucose $\times$ fasting serum insulin $/ 22.5$.

\subsection{Pathological Morphology of the Liver}

Treatment of liver tissue was carried out according to a previous study [18]. The same part of each mouse liver sample was collected, fixed, dehydrated, embedded, cut into $\sim 5 \mu \mathrm{m}$ thick slices, and finally stained with $\mathrm{H} \& \mathrm{E}$. The liver micrographs were evaluated by the SAF scoring system [19].

\subsection{Determination of Cytokines in the Liver}

Liver tissue samples of $150 \mathrm{mg}$ were crushed with a buffer solution to prepare the liver homogenate, then centrifuged $\left(10,000 \times g, 15 \mathrm{~min}, 4^{\circ} \mathrm{C}\right)$ to collect the supernatant. Next, three cytokines (TNF- $\alpha$, IL-10, IL-6) were determined by the commercialized ELISA kit (R\&D Systems, Minneapolis, MN, USA) and the protein concentration was determined with a commercialized kit (Beyotime Biotechnology, Shanghai, China).

\subsection{The Analysis of the SCFAs in Feces}

Analysis of short chain fatty acids (SCFAs) were performed as previously described [20]. The feces stored at $-80{ }^{\circ} \mathrm{C}$ were taken out, lyophilized, weighed and put into a sterile centrifuge tube, then mixed with $500 \mu \mathrm{L}$ saturated sodium chloride solution and soaked for $30 \mathrm{~min}$. Next, $40 \mu \mathrm{L}$ of $10 \%(v / v) \mathrm{H}_{2} \mathrm{SO}_{4}$ was added to acidify the samples to make 
them fully dissolved. After acidification, the samples were homogenized with $1 \mathrm{~mL}$ ethyl ether and centrifuged $\left(4^{\circ} \mathrm{C}, 13,000 \times g, 15 \mathrm{~min}\right)$. Then, the supernatant was removed to a clean tube with $0.25 \mathrm{~g}$ anhydrous sodium sulfate in advance and stood for $15 \mathrm{~min}$ to absorb water. After centrifugation and filtration, it was analysed by GC-MS (GCMS-QP2010 Ultra system, Shimadzu Corporation, Kyoto, Japan) as previously described [21].

\subsection{Analysis of the Gut Microbiota}

Fecal genomic DNA was extracted with FastDNA SPIN Kit for Feces (MP Biomedicals, Santa Ana, CA, USA), and then amplified, purified, quantified and mixed with equal quality samples [22]. Library preparation, library testing, and MISeq platform PE300 sequencing were performed as previously described [23]. After sequencing, the QIIME2 pipeline was used to analyze the deplaning data [24]. Linear discriminant analysis effect size (LEfSe) analyses were performed to identify the specific taxa on the MicrobiomeAnalyst online platform [25]. The indexess of Chao 1 and Simpson were used to reflect the alpha diversity of the gut microbiota. Additionally, the beta diversity of gut microbiota was assessed for differences in species composition using the principal coordinate analysis (PCoA) method and visualization [26].

\subsection{Statistical Analysis}

Data were presented using mean \pm SEM. The statistical assessments among the six groups were calculated by a one-way ANOVA with Tukey's post-hoc test (parametric analysis) or the Kruskal-Wallis test followed by Duncan's post-hoc test (non-parametric analysis). A $p$-value $<0.05$ was defined as statistically significant.

\section{Results}

\subsection{Effect of L. reuteri on Body Weight, Food Intake and Fat Index}

The pathological feature of MS is central obesity, which is primarily manifested by changes in body weight, dietary intake and fat proportion. At the third week of the animal trial, body weight differences were significantly different between NC and HFD groups $(p<0.05)$, but not different in other groups (Figure 1A). Before ending the trial, the body weight of the mice which were fed with different diets in the HFD and NC groups increased by $57.30 \%$ and $23.33 \%$, respectively (Figure $1 \mathrm{~B}$ ). In addition, the daily feed intake and energy conversion efficiency in the HFD-fed mice were higher than those in the NC mice during the whole experiment period, which also led to a higher proportion of epididymal fat and a lower proportion of brown fat in the HFD-fed mice (Figure 1C-F). After interventions with $L$. reuteri for 14 weeks, the food intake and energy conversion rate were significantly decreased $(p<0.05)$, and in particular, the epididymal fat proportion in the L. reuteri FYNLJ109L1 treated mice was significantly decreased $(p<0.01)$, whereas the brown fat index was increased but without significance.

\subsection{Effect of L. reuteri on Serum Lipid, Glucose and Insulin}

Serum LDL-C, HDL-C, LDL-C/HDL-C and TC in the mice with a long-term intake of an HFD were significantly higher than those in the control mice $(p<0.01)$, but not for serum TG (Figure 2A-E). Serum LDL-C and LDL-C/HDL-C were significantly decreased after $L$. reuteri intervention $(p<0.01)$. 
A

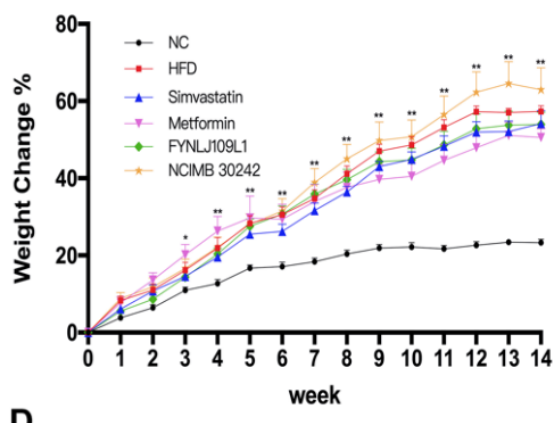

D

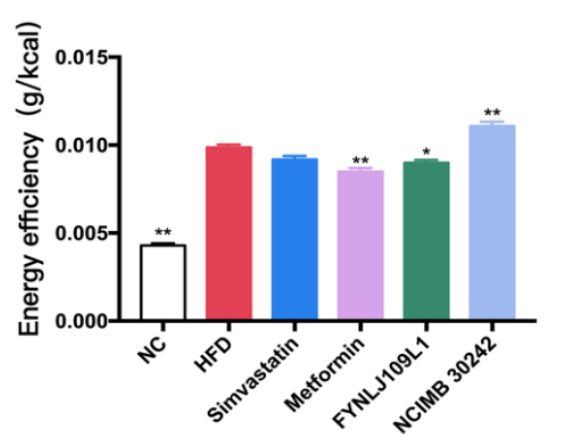

B
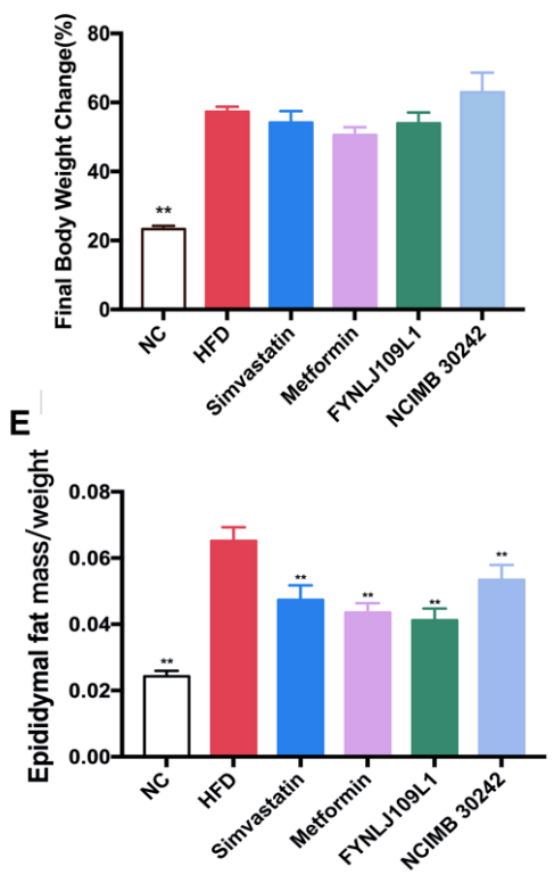

C
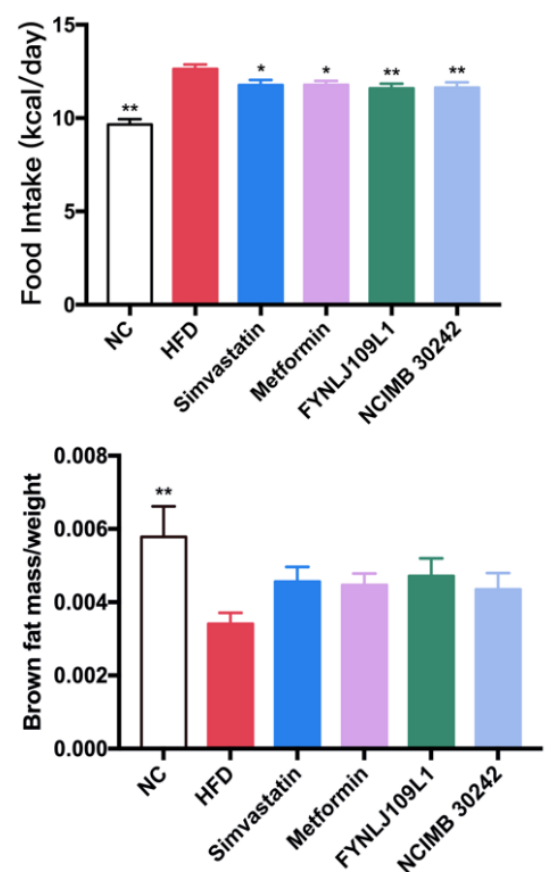

Figure 1. Effects of L. reuteri intervention on body weight, food intake and fat ratio. (A) Body weight. The $t$-test was only performed between NC and HFD, ${ }^{*} p<0.05$ and ${ }^{* *} p<0.01$; (B) Final body weight change; (C) Food intake; (D) Energy efficiency; (E) Adipose mass /weight ratio; (F) Ratio of brown fat to weight. $\left(n=8 .{ }^{*} p<0.05\right.$ and ${ }^{* *} p<0.01$ for comparison with HFD).

A

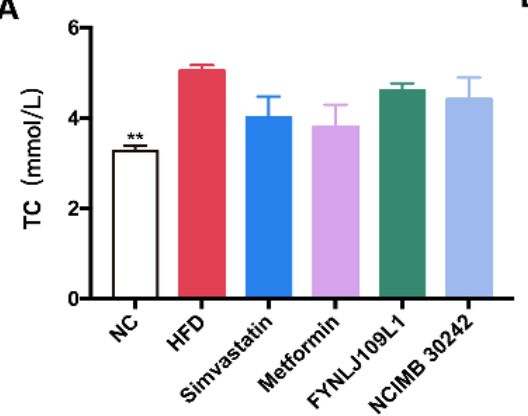

D

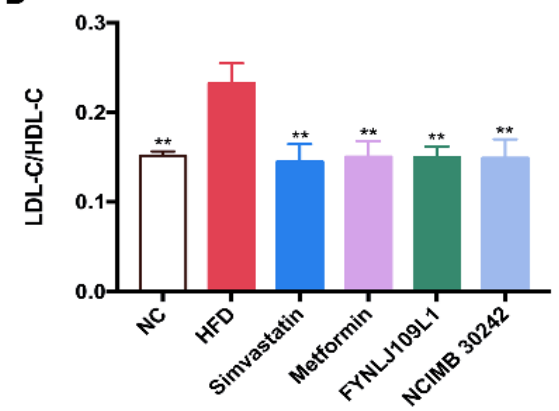

B

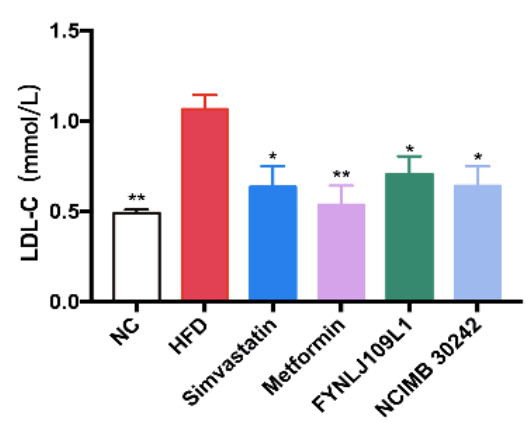

E

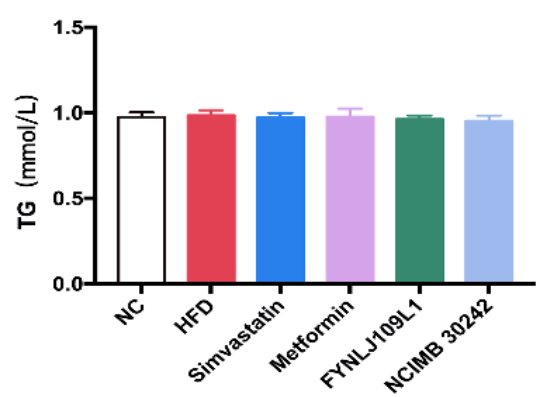

C

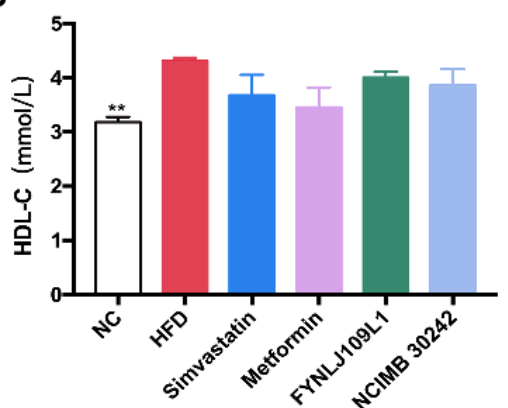

Figure 2. Effects of L. reuteri intervention on serum lipid levels. (A) TC; (B) LDL-C; (C) HDL-C; (D) LDL-C/HDL-C ratio; (E) TG. $\left(n=8 .{ }^{*} p<0.05\right.$ and ${ }^{* *} p<0.01$ for comparison with HFD.).

At the end of the experiment, high-fasting blood glucose (Figure 3A), high-fasting insulin (Figure 3B), the onset of insulin resistance (Figure 3C) and blood glucose recovered slowly after an OGTT (Figure 3D), and high AUCglucose values (Figure 3E) were found 
in the HFD group. L. reuteri FYNLJ109L1 treatment could moderately reduce the fasting insulin $(p<0.05)$, and significantly increase the clearance of glucose clusters during the OGTT period $(p<0.01)$, which was superior to L. reuteri NCIMB 30242.

A

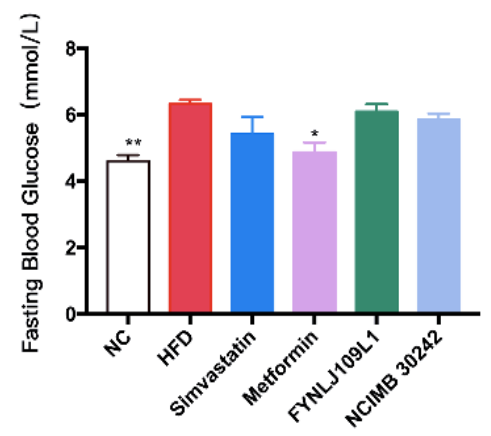

D

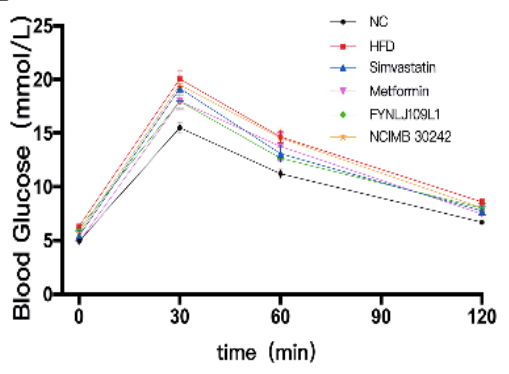

B

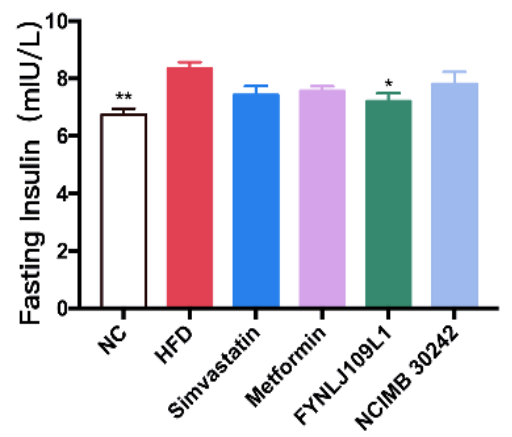

E

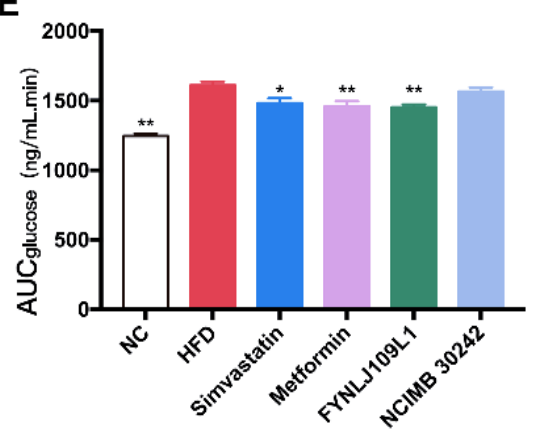

C

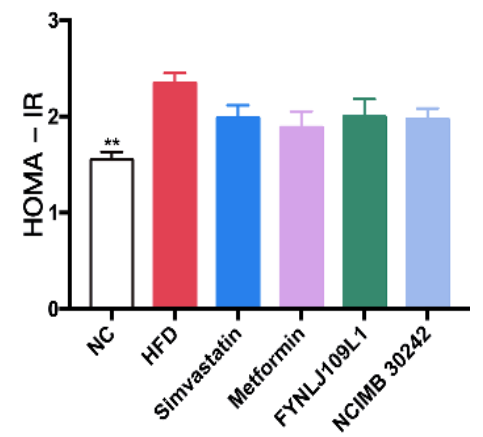

Figure 3. Effects of L. reuteri intervention on serum glucose and insulin levels. (A) Fasting blood glucose; (B) Fasting insulin; (C) HOMA-IR; (D) Blood glucose before and after the oral glucose; (E) AUC of the OGGT. NC, normal control; HFD, high-fat diet. Data are represented as mean $\pm \operatorname{SEM}(n=8) .{ }^{*} p<0.05$ and ${ }^{* *} p<0.01$ for comparison with HFD.

\subsection{Effect of L. reuteri on Liver Injury}

In the HFD group, the results were consistent with vacuolar degeneration of hepatocytes, the presence of lipid droplets accounting for $30-50 \%$ of the field of vision, and infiltration of inflammatory cells (Figure 4A,B). Regarding the level of liver inflammation, compared to the control mice, the concentrations of TNF- $\alpha$ and IL-6 in the HFD-fed mice were significantly increased, whereas the concentration of IL-10 was significantly decreased $(p<0.01)$ compared with that in the control group (Figure 4C-E). Histopathological scores showed that the intervention of L. reuteri FYNLJ109L1 significantly reduced the liver lesions $(p<0.01)$. In addition, compared with the HFD group, TNF- $\alpha(p<0.001)$ and IL-6 $(p<0.01)$ in the L. reuteri FYNLJ109L1 treated mice decreased significantly, respectively. Moreover, the concentration of IL-10 in the L. reuteri FYNLJ109L1 group was 1.82 times higher than that in the HFD group $(p<0.01)$, which was similar to that in the NC mice.

\subsection{Effect of L. reuteri on SCFAs}

There were differences in SCFAs in the feces of mice fed with the different diets. The HFD resulted in a decrease in SCFAs in the feces, and acetic, propionic and butyric acids were significantly decreased to $49.24 \%(p<0.001), 23.90 \%(p<0.05)$ and $24.01 \%(p<0.05)$, respectively (Figure 5A-C). Moreover, no significant change was found for those three acids in those mice intervented with $L$. reuteri strains, except propionic acid in the $L$. reuteri NCIMB 30,242 $(p<0.01)$. 
A

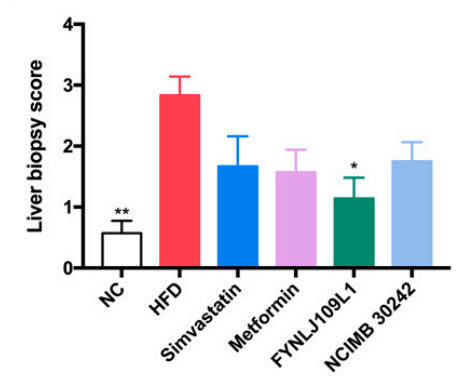

C

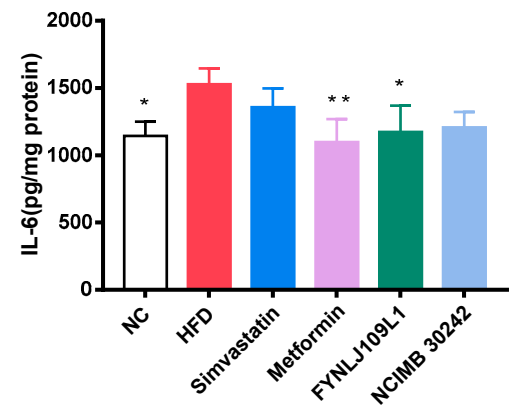

B

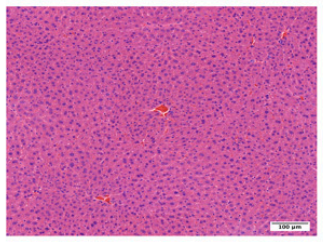

NC

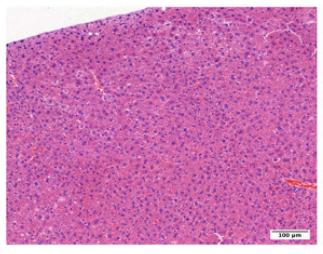

Metformin

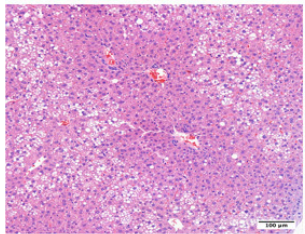

HFD

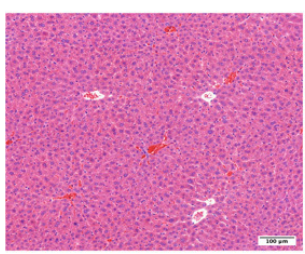

FYNLJ109L1

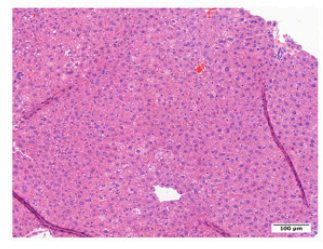

Simvastatin

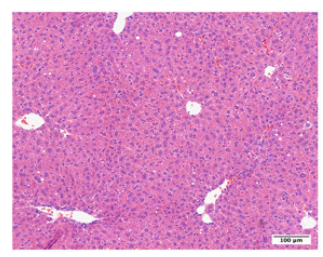

NCIMB 30242
D

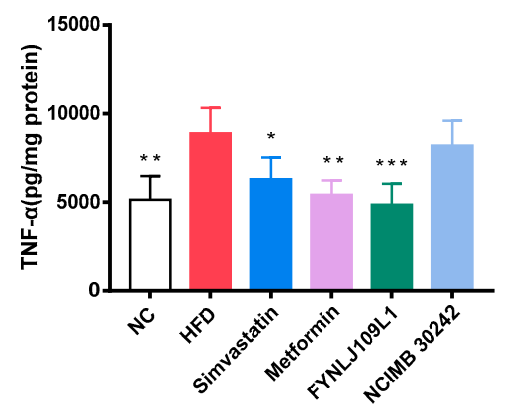

$\mathbf{E}$

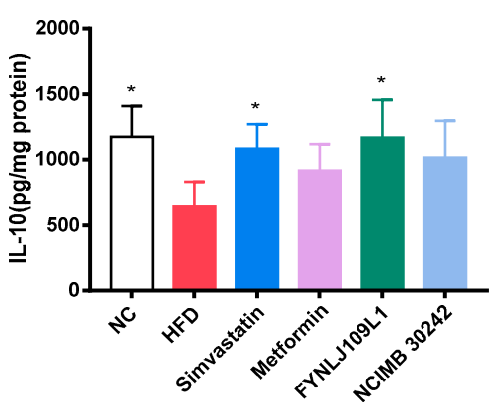

Figure 4. Effects of L. reuteri intervention on liver injury. (A) Liver tissue pathology score; (B) Representative pictures of H\&E-stained liver sections from indicated mice (final magnification, 200×); (C) Liver IL-6 concentration; (D) Liver TNF- $\alpha$ concentration; (E) Liver IL-10 concentration.NC, normal control; HFD, high-fat diet. Data are represented as mean \pm SEM $(n=8) .{ }^{*} p<0.05,{ }^{* *} p<0.01$ and ${ }^{* * *} p<0.001$ for comparison with HFD.

A

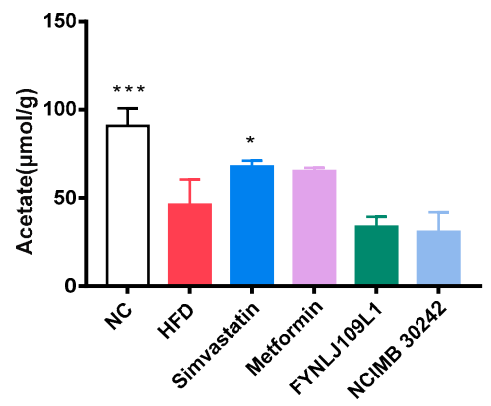

B

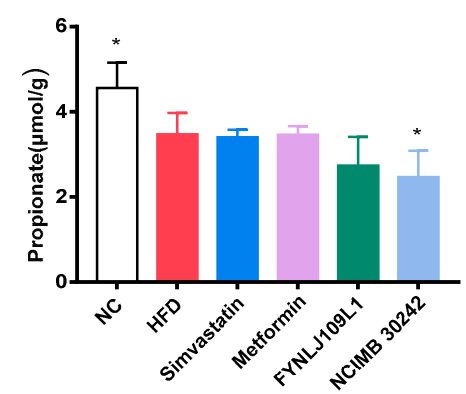

C

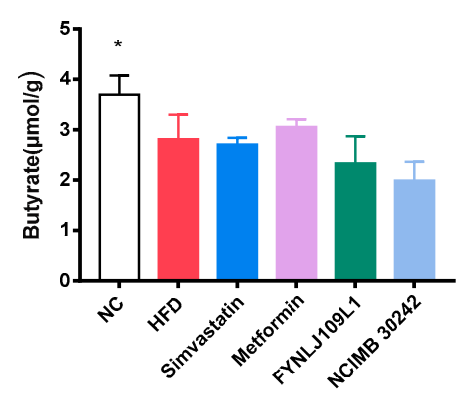

Figure 5. Effects of L. reuteri intervention on fecal SCFAs. (A) Acetic acid; (B) Propionic acid; (C) Butyric acid. NC, normal control; HFD, high-fat diet. Data are represented as mean $\pm \operatorname{SEM}(n=8) .{ }^{*} p<0.05$ and ${ }^{* * *} p<0.001$ for comparison with HFD.

\subsection{Modulation of Gut Microbiota by L. reuteri}

Both diet and L. reuteri interventions could affect the gut microbiota. Amplicon sequencing was performed to analyze the changes in the gut microbiota in terms of diversity and composition. Chao1, which represents a species abundance, and Simpson index, which represents the microbial diversity, were used to analyze the diversity of the gut microbiota among groups. No difference was found in the Chao1 and Simpson indexes between the HFD and NC groups, indicating that the HFD had no influence on the gut microbiota diversity (Figure 6A,B). Both the L. reuteri strains used for the intervention also did not significantly affect the gut microbiota diversity. Beta diversity reflected in the PCoA 
analysis based on the Bray-Curtis distance indicated that there was a significant bacterial community between the NC group on the normal control diet and the other groups with an HFD (Figure 6C). Notably, 14-weeks of L. reuteri treatments in MS mice resulted in a partial normalization of intestinal microbial diversity in PCoA1 (25.70\%) or PCoA2 (15.95\%).

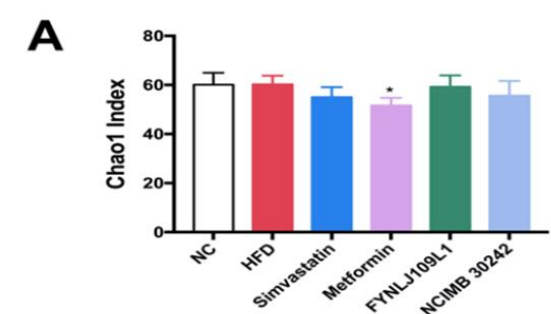

C

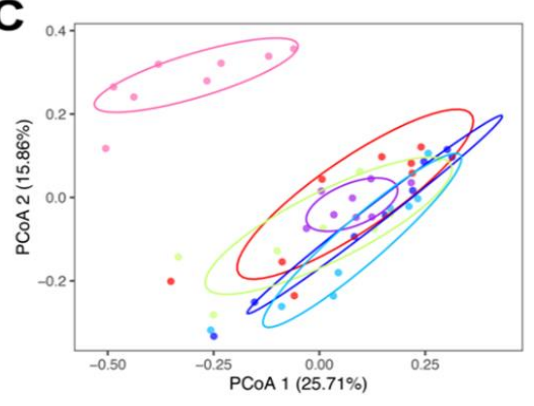

E
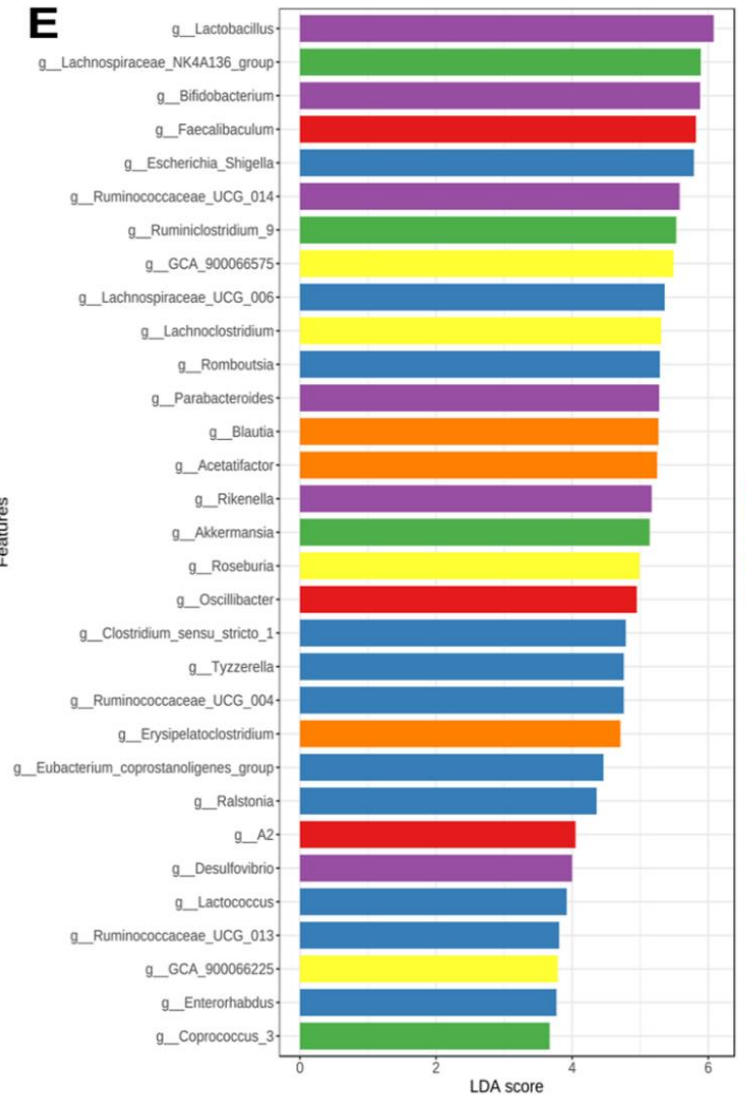

B

D
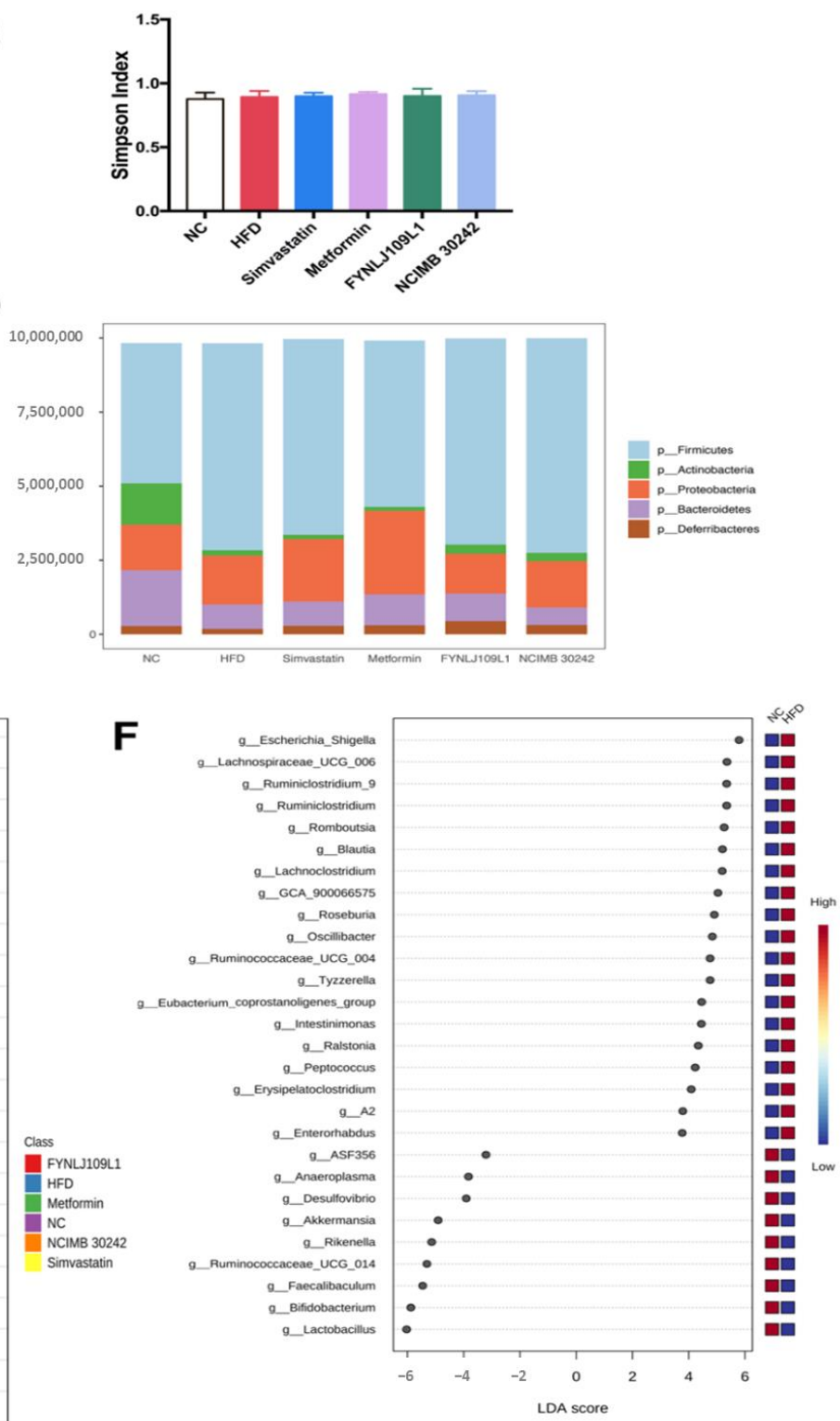

G

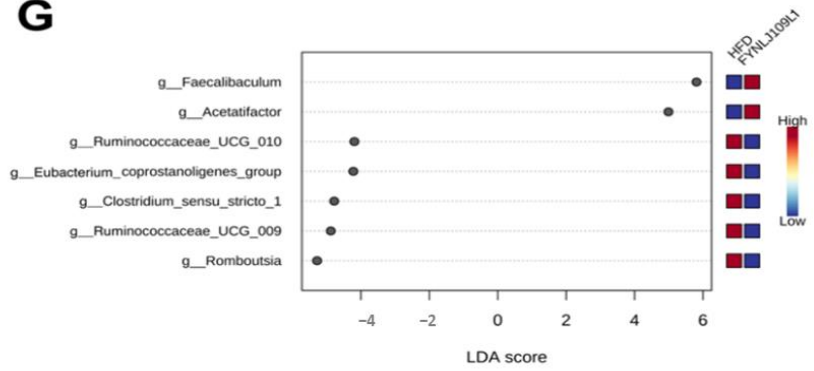

Figure 6. The gut microbiota modulation by the L. reuteri intervention. (A) Chao1 index; (B) Simpson index; (C) PCoA; (D) Microbial distribution at the phylum level; (E) LEfSe analysis of the gut microbiota (LDA value > 3.0); (F) LEfSe analysis between the NC and HFD (LDA value > 3.0); (G) LEfSe analysis between the HFD and FYNLJ109L1 (LDA value > 3.0). $\left(n=8 .{ }^{*} p<0.05\right.$ for comparison with the HFD). 
The profile of the gut microbiota changed with the different diets. At the phylum level, firmicutes $(47.33 \%)$ and bacteroidetes $(18.79 \%)$ accounted for the largest proportion in the normal control group, followed by proteobacteria $(15.40 \%)$ and actinobacteria (13.94\%). After a 14-week intake of the HFD, the proportions of the four major bacteria changed, and the relative abundance of firmicutes and proteobacteria raised to $69.85 \%$ and $16.49 \%$, respectively, whereas the relative abundance of bacteroidetes and actinobacteria were reduced to $8.24 \%$ and $1.81 \%$, respectively (Figure $6 \mathrm{D}$ ). Compared with the control mice, the ratio of bacteroides to firmicutes associated with obesity in the HFD group decreased significantly $(p<0.01)$, and the increasing trend could be reversed with treatment of each $L$. reuteri strain. With the LEfSe analysis, the species with significant differences in abundance among the groups were mainly Lactobacillus, Bifidobacterium, Ruminococcaceae UCG_014, Parabacteroides, Rikenella and Desulfovibrio in the control group, whereas Escherichia_Shigella, Clostridium_sensu_stricto_1, Lachnospiraceae UCG_006, Romboutsia, Tyzzerella, Ruminococcaceae UCG_004, Ralstonia, Lactococcus, Ruminococcaceae UCG_013, Eubacterium_coprostanoligenes_group and Enterorhabdus were enriched in the HFD group. Moreover, Faecalibaculum, Oscillibacter and A2 were the dominant genus in the L. reuteri FYNLJ109L1-treated mice (Figure 6E). Compared with the NC mice, the gut microbiota of the mice with a 14-week intake of an HFD showed an increased abundance of 19 genera and decreased the abundance of 9 genera (Figure 6F). In addition, treatments with L. reuteri affected 7 genera, which were Faecalibaculum, Eubacterium_coprostanoligenes_group, Acetatifactor, Ruminococcaceae_UCG_009, Ruminococcaceae_UCG_010,Clostridium_sensu_stricto_1 and Romboutsia (Figure 6G).

\section{Discussion}

MS and a series of related diseases are among the global human health issues. In recent years, regulating the gut microbiota by taking probiotics has become a new approach to alleviating MS. Additionally, there is more evidence that gut microbes can produce molecules to regulate the appetite and satiety that play key roles in energy conversion and reducing the accumulation of adipose tissue [27]. For example, administration of Lactobacillus fermented milk in Wistar rats was shown to regulate the production of the satiety gratitude hormone [28]. Our results also confirmed that both L. reuteri interventions significantly decreased the daily feed intake, energy conversion efficiency and epididymal fat proportion [29]. Furthermore, the L. reuteri intervention in the mice fed with an HFD significantly reduced the concentration of LDL-C and the ratio of LDL-C/HDL-C, suggesting that the L. reuteri intervention could descrase the risk of coronary disease [30] and its related metabolic syndrome to a certain extent. Moreover, between the two strains analyzed, the mice that were fed with an HFD and treated with L. reuteri FYNLJ109L1 showed weaker MS symptoms, which may be due to the bacterial surface substance [31], gut microbiota modulation [32] and attenuate inflammation [33].

The liver manifestation of MS is NAFLD, which is the target organ damage caused by the disorder of blood glucose and lipid metabolism. A fatty liver occurs in patients with high body fat if the liver fat accumulation is excessive, which can be alleviated and improved through early detection and treatment [9]. NAFLD in the mice induced by a high-fructose diet can be protected by a L. rhamnosus GG intervention [34], and our results also reached a similar conclusion. Even the high-temperature inactivated L. reuteri GMNL-263 can reduce the fatty liver syndrome in HFD-fed hamsters, reduce the degree of liver fibrosis, and decrease the serum LDL-C and plasma malondialdehyde [31]. Therefore, intervention with L. reuteri FYNLJ109L1 nor L. reuteri NCIMB 30,242 alleviated those liver injury symptoms, which may be responsible for the potentially beneficial substances in the strain and needs to be verified by further high-temperature killing inactivation.

MS and related diseases can cause biological disorders such as adverse changes in intestinal bacteria [35]. Previous reports have shown that probiotic interventions can alleviate MS by regulating the gut microbiota and even rebalance those damaged by an HFD [12,33]. Among the two largest bacterial phyla with a great difference between obese 
and lean individuals, obese subjects had a highly relative abundance of firmicutes [36], whereas thin subjects had greater bacteroidetes in the gut, resulting in weight loss [37], which was consistent with our results. An increased abundance in firmicutes and a decreased abundance in bacteroides can lead to an increase in heat and obesity [4]. Reducing the ratio of firmicutes/bacteroidetes could reduce the energy acquisition and may have potential benefits for preventing fatty liver disease [4]. In fact, our results were in line with this, which showed with a lower ratio of firmicutes/bacteroidetes in the control compared with the HFD-fed mice and virtually no areas of steatosis in liver tissues. For the other phylum, proteobacteria represent microbial biomarkers for imbalanced gut microbial communities [38] and actinobacteria are positively associated with dietary fat intake [39]. Furthermore, in accordance with our results, proteobacteria increased in the HFD group, and it returned to normal in the mice treated with probiotics. The fact that the abundance of actinobacteria decreased in the HFD group, but increased in the control group and L. reuteri FYNLJ109L1 group, was puzzling. Thus, the effective intervention effect of L. reuteri may be due to the correlative effect of gut microbiota, but the analysis of detailed taxa still needs to be performed.

At the genus level, a long-term HFD resulted in changes in the species and abundance of the host intestinal microbes. Among the genus altered significantly, romboutsia is sensitive to bile salt and associated with energy metabolism, making it a candidate for predicting and treating the metabolic diseases [40]. Prior research showed that clostridium spp. is the causative agent of intestinal disease in goats [41], suggesting that clostridium has an adverse effect on the intestinal barrier [42]. Therefore, the accumulation of clostridium sensu stricto-1 in the gut may be responsible for the aggravation of MS symptoms in mice. The decreased abundance of these bacteria may be one of the reasons for the protective effects of L. reuteri FYNLJ109L1 on atherosclerosis and dyslipidemia. Moreover, acetatifactor was increased in mice fed with an HFD and treated with tea extracts [29]. Compared with the NC group, the abundance of faecalibaculum in the HFD group showed a lower relative abundance [43], and the reduced abundance of ruminococcaceae is conducive to intestinal homeostasis [44]. The reduction of SCFAs producers such as ruminococcaceae, eubacterium and faecalibacterium may lead to the reduction in SCFAs in L. reuteri FYNLJ109L1 treated mice. The previous literature has reported that the increase in SCFAs may alleviate the metabolic syndrome; however, even if SCFAs production is reduced, other metabolites may also be involved, such as indoles, produced by L. reuteri from tryptophan, which have been reported to relieve MS [1]. Therefore, at the genus level, further analysis of gut microbiota composition showed the L. reuteri FYNLJ109L1 intervention could effectively regulate several key taxa of an imbalanced gut microbiota with a long-term high-fat diet, and was also one of the reasons for improving MS.

In addition, an abnormal gut microbiota can promote inflammation, resulting in a state of low-grade systemic inflammation [45]. The balance of the gut microbiota is important for maintaining the normal physiological function of the intestinal barrier [46], which may otherwise lead to an increased gut permeability and LPS translocation, resulting in a subclinical proinflammatory state [47]. When endotoxins produced by the abnormal intestinal microorganisms induced TLR- 4 activation by altering the gut-liver axis, this, in turn, induced the pro-inflammatory responses by activating IL- 6 and TNF- $\alpha$ in the liver [48]. It is well known that IL-6 is one of the typical cytokines associated with insulin resistance. In particular, it can induce insulin resistance by a phosphorylation reduction of insulin receptor substrates or transcription inhibition [49]. TNF- $\alpha$ is an important mediator for insulin resistance in obese subjects, and it mainly induces insulin resistance by weakening the insulin receptor signal transduction [50]. Furthermore, the elevated TNF- $\alpha$ levels can reduce the lipid oxidation and increase the lipid accumulation, leading to obesity [51]. The main advantage of probiotics is that they can reduce the inflammation by altering the gut microbiota and alleviating the associated pathological conditions. It has been shown that some Lactobacillus secrete soluble molecules that inhibit the production of TNF- $\alpha$ via activating the mononuclear macrophages [52]. In our study, it was found that 
the long-term HFD increased the IL- 6 and TNF- $\alpha$, which were typical pro-inflammatory cytokines; moreover, administration of $L$. reuteri FYNLJ109L1 significantly inhibited the increase in TNF- $\alpha$ and IL-6. In addition, IL-10 is a typical anti-inflammatory cytokine which could prevent the insulin resistance and MS in mice [53]. An increase in IL-10 in probiotictreated mice with a HFD may also contribute to ameliorating the metabolic changes, which is consistent with previous findings, connecting the changes of intestinal microbial composition with a reduction in low-grade inflammation and metabolic dysfunction [54].

\section{Conclusions}

L. reuteri showed a strain-dependent protective effect on metabolic disorders. L. reuteri FYNLJ109L1 may reduce weight gain, fat accumulation, insulin resistance, dyslipidemia, and liver tissue damage by modulating the gut microbiota (specifically via a decrease in romboutsia and clostridium sensu stricto- 1 and an increase in acetatifactor).

Author Contributions: Conceptualization, B.Y. and H.Z.; methodology, B.Y. and F.Z.; software, B.Y.; validation, B.Y., F.Z. and J.Z.; formal analysis, B.Y. and F.Z.; investigation, B.Y. and F.Z.; resources, H.Z.; data curation, B.Y., F.Z. and J.Z.; writing-original draft preparation, B.Y. and F.Z.; writing-review and editing, C.S., R.P.R., H.Z. and W.C.; visualization, B.Y.; supervision, H.Z.; project administration, H.Z.; funding acquisition, W.C. All authors have read and agreed to the published version of the manuscript.

Funding: This research was supported by the National Natural Science Foundation of China (Nos. 32021005, 31820103010), 111 project (BP0719028), and Collaborative Innovation Center of Food Safety and Quality Control in Jiangsu Province.

Institutional Review Board Statement: The study was conducted according to the guidelines of the Declaration of Helsinki, and approved by the Animal Ethics Committee of Jiangnan University (JN. No20200630c1201130(121)).

Conflicts of Interest: The authors declare no conflict of interest.

\section{References}

1. Natividad, J.M.; Agus, A.; Planchais, J.; Lamas, B.; Jarry, A.C.; Martin, R.; Michel, M.L.; Chong-Nguyen, C.; Roussel, R.; Straube, M.; et al. Impaired aryl hydrocarbon receptor ligand production by the gut Microbiota is a key factor in metabolic syndrome. Cell Metab. 2018, 28, 737-749. [CrossRef]

2. Kaur, J. A comprehensive review on metabolic syndrome. Cardiol. Res. Pract. 2014, 2014, 943162. [CrossRef]

3. Power, E.E.; Johnson, J.R.; Clouston, A.D. Metabolic factors and non-alcoholic fatty liver disease as co-factors in other liver diseases. Dig. Dis. 2010, 28, 186-191. [CrossRef]

4. Turnbaugh, P.J.; Ley, R.E.; Mahowald, M.A.; Magrini, V.; Mardis, E.R.; Gordon, J.I. An obesity-associated gut microbiome with increased capacity for energy harvest. Nature 2006, 444, 1027-1031. [CrossRef]

5. Zhu, K.; Tan, F.; Mu, J.; Yi, R.; Zhou, X.; Zhao, X. Anti-obesity effects of Lactobacillus fermentum CQPC05 isolated from Sichuan pickle in high-fat diet-induced obese mice through PPAR- $\alpha$ signaling pathway. Microorganisms 2019, 7, 194. [CrossRef] [PubMed]

6. Wang, J.; Qin, J.; Li, Y.; Cai, Z.; Li, S.; Zhu, J.; Zhang, F.; Liang, S.; Zhang, W.; Guan, Y.; et al. A metagenome-wide association study of gut microbiota in type 2 diabetes. Nature 2012, 490, 55-60. [CrossRef]

7. Vrieze, A.; Van Nood, E.; Holleman, F.; Salojärvi, J.; Kootte, R.S.; Bartelsman, J.F.W.M.; Dallinga-Thie, G.M.; Ackermans, M.T.; Serlie, M.J.; Oozeer, R.; et al. Transfer of intestinal microbiota from lean donors increases insulin sensitivity in individuals with metabolic syndrome. Gastroenterology 2012, 143, 913-916. [CrossRef] [PubMed]

8. Zhang, F.; Qiu, L.; Xu, X.; Liu, Z.; Zhan, H.; Tao, X.; Shah, N.P.; Wei, H. Beneficial effects of probiotic cholesterol-lowering strain of Enterococcus faecium WEFA23 from infants on diet-induced metabolic syndrome in rats. J. Dairy Sci. 2017, 100, 1618-1628. [CrossRef]

9. Iqbal, U.H.; Westfall, S.; Prakash, S. Novel microencapsulated probiotic blend for use in metabolic syndrome: Design and in-vivo analysis. Artif. Cells Nanomed. Biotechnol. 2018, 46, S116-S124. [CrossRef] [PubMed]

10. Kim, K.A.; Jeong, J.J.; Kim, D.H. Lactobacillus brevis OK56 ameliorates high-fat diet-induced obesity in mice by inhibiting NF- $\mathrm{B}$ activation and gut microbial LPS production. J. Funct. Foods 2015, 13, 183-191. [CrossRef]

11. Yadav, H.; Lee, J.H.; Lloyd, J.; Walter, P.; Rane, S.G. Beneficial metabolic effects of a probiotic via butyrate-induced GLP-1 hormone secretion. J. Biol. Chem. 2013, 288, 25088-25097. [CrossRef]

12. Wang, J.; Tang, H.; Zhang, C.; Zhao, Y.; Derrien, M.; Rocher, E.; Van-Hylckama Vlieg, J.E.T.; Strissel, K.; Zhao, L.; Obin, M.; et al. Modulation of gut microbiota during probiotic-mediated attenuation of metabolic syndrome in high fat diet-fed mice. ISME J. 2015, 9, 1-15. [CrossRef] 
13. Hsieh, F.C.; Lee, C.L.; Chai, C.Y.; Chen, W.T.; Lu, Y.C.; Wu, C.S. Oral administration of Lactobacillus reuteri GMNL-263 improves insulin resistance and ameliorates hepatic steatosis in high fructose-fed rats. Nutr. Metab. 2013, 10, 35. [CrossRef] [PubMed]

14. Taranto, M.P.; Medici, M.; Perdigon, G.; Ruiz Holgado, A.P.; Valdez, G.F. Evidence for hypocholesterolemic effect of Lactobacillus reuteri in hypercholesterolemic mice. J. Dairy Sci. 1998, 81, 2336-2340. [CrossRef]

15. Fåk, F.; Bäckhed, F. Lactobacillus reuteri prevents diet-induced obesity, but not atherosclerosis, in a strain dependent fashion in Apoe $^{-/-}$Mice. PLoS ONE 2012, 7, e46837. [CrossRef] [PubMed]

16. Jiang, J.; Feng, N.; Zhang, C.; Liu, F.; Zhao, J.; Zhang, H.; Zhai, Q.; Chen, W. Lactobacillus reuteri A9 and Lactobacillus mucosae A13 isolated from Chinese superlongevity people modulate lipid metabolism in a hypercholesterolemia rat model. FEMS Microbiol. Lett. 2020, 366, fnz254. [CrossRef] [PubMed]

17. Jones, M.L.; Martoni, C.J.; Parent, M.; Prakash, S. Cholesterol-lowering efficacy of a microencapsulated bile salt hydrolase-active Lactobacillus reuteri NCIMB 30242 yoghurt formulation in hypercholesterolaemic adults. Br. J. Nutr. 2012, 107, 1505-1513. [CrossRef]

18. Wang, G.; Jiao, T.; Xu, Y.; Li, D.; Si, Q.; Hao, J.; Zhao, J.; Zhang, H.; Chen, W. Bifidobacterium adolescentis and Lactobacillus rhamnosus alleviate non-alcoholic fatty liver disease induced by a high-fat, high-cholesterol diet through modulation of different gut microbiota-dependent pathways. Food Funct. 2020, 11, 6115-6127. [CrossRef]

19. Bedossa, P.; Burt, A.A.; Gouw, A.H.A.; Lackner, C.; Schirmacher, P.; Terracciano, L.; Tiniakos, D.; Brain, J.; Bury, Y.; Cabibi, D.; et al. Utility and appropriateness of the fatty liver inhibition of progression (FLIP) algorithm and steatosis, activity, and fibrosis (SAF) score in the evaluation of biopsies of nonalcoholic fatty liver disease. Hepatology 2014, 60, 565-575. [CrossRef]

20. Wang, L.; Hu, L.; Yan, S.; Jiang, T.; Fang, S.; Wang, G.; Zhao, J.; Zhang, H.; Chen, W. Effects of different oligosaccharides at various dosages on the composition of gut microbiota and short-chain fatty acids in mice with constipation. Food Funct. 2017, 8, 1966-1978. [CrossRef]

21. Zhu, G.; Ma, F.; Wang, G.; Wang, Y.; Zhao, J.; Zhang, H.; Chen, W. Bifidobacteria attenuate the development of metabolic disorders, with inter- and intra-species differences. Food Funct. 2018, 9, 3509-3522. [CrossRef]

22. Zhao, L.; Wang, G.; Siegel, P.; He, C.; Wang, H.; Zhao, W.; Zhai, Z.; Tian, F.; Zhao, J.; Zhang, H.; et al. Quantitative genetic background of the host influences gut microbiomes in chickens. Sci. Rep. 2013, 3, 1163. [CrossRef] [PubMed]

23. Chen, Y.; Jin, Y.; Stanton, C.; Paul Ross, R.; Zhao, J.; Zhang, H.; Yang, B.; Chen, W. Alleviation effects of Bifidobacterium breve on DSS-induced colitis depends on intestinal tract barrier maintenance and gut microbiota modulation. Eur. J. Nutr. 2020, 60, 369-387. [CrossRef] [PubMed]

24. Caporaso, J.G.; Kuczynski, J.; Stombaugh, J.; Bittinger, K.; Bushman, F.D.; Costello, E.K.; Fierer, N.; Pẽa, A.G.; Goodrich, J.K.; Gordon, J.I.; et al. QIIME allows analysis of high-throughput community sequencing data. Nat. Methods 2010, 7, 335-336. [CrossRef]

25. Dhariwal, A.; Chong, J.; Habib, S.; King, I.L.; Agellon, L.B.; Xia, J. MicrobiomeAnalyst: A web-based tool for comprehensive statistical, visual and meta-analysis of microbiome data. Nucleic Acids Res. 2017, 45, W180-W188. [CrossRef] [PubMed]

26. Mao, B.; Gu, J.; Li, D.; Cui, S.; Zhao, J.; Zhang, H.; Chen, W. Effects of different doses of fructooligosaccharides (FOS) on the composition of mice fecal microbiota, especially the bifidobacterium composition. Nutrients 2018, 10, 1105. [CrossRef] [PubMed]

27. Greiner, T.; Bäckhed, F. Effects of the gut microbiota on obesity and glucose homeostasis. Trends Endocrinol. Metab. 2011, 22, 117-123. [CrossRef] [PubMed]

28. Forssten, S.D.; Korczyńska, M.Z.; Zwijsen, R.M.L.; Noordman, W.H.; Madetoja, M.; Ouwehand, A.C. Changes in satiety hormone concentrations and feed intake in rats in response to lactic acid bacteria. Appetite 2013, 71, 16-21. [CrossRef]

29. Liu, J.; Hao, W.; He, Z.; Kwek, E.; Zhao, Y.; Zhu, H.; Liang, N.; Ma, K.Y.; Lei, L.; He, W.-S.; et al. Beneficial effects of tea water extracts on the body weight and gut microbiota in C57BL/6J mice fed with a high-fat diet. Food Funct. 2019, 10, $2847-2860$. [CrossRef] [PubMed]

30. Costabile, A.; Buttarazzi, I.; Kolida, S.; Quercia, S.; Baldini, J.; Swann, J.R.; Brigidi, P.; Gibson, G.R. An in vivo assessment of the cholesterol-lowering efficacy of Lactobacillus plantarum ECGC 13110402 in normal to mildly hypercholesterolaemic adults. PLoS ONE 2017, 12, e0187964. [CrossRef] [PubMed]

31. Ting, W.J.; Kuo, W.W.; Hsieh, D.J.Y.; Yeh, Y.L.; Day, C.H.; Chen, Y.H.; Chen, R.J.; Padma, V.V.; Chen, Y.H.; Huang, C.Y. Heat killed lactobacillus reuteri GMNL-263 reduces fibrosis effects on the liver and heart in high fat diet-hamsters via TGF- $\beta$ suppression. Int. J. Mol. Sci. 2015, 16, 25881-25896. [CrossRef]

32. Lecomte, V.; Kaakoush, N.O.; Maloney, C.A.; Raipuria, M.; Huinao, K.D.; Mitchell, H.M.; Morris, M.J. Changes in gut microbiota in rats fed a high fat diet correlate with obesity-associated metabolic parameters. PLoS ONE 2015, 10, e0126931. [CrossRef]

33. Qiao, Y.; Sun, J.; Xia, S.; Li, L.; Li, Y.; Wang, P.; Shi, Y.; Le, G. Effects of different Lactobacillus reuteri on inflammatory and fat storage in high-fat diet-induced obesity mice model. J. Funct. Foods 2015, 14, 424-434. [CrossRef]

34. Ritze, Y.; Bárdos, G.; Claus, A.; Ehrmann, V.; Bergheim, I.; Schwiertz, A.; Bischoff, S.C. Lactobacillus rhamnosus GG Protects against non-alcoholic fatty liver disease in mice. PLoS ONE 2014, 9, e80169. [CrossRef] [PubMed]

35. Creely, S.J.; McTernan, P.G.; Kusminski, C.M.; Fisher, F.M.; Da Silva, N.F.; Khanolkar, M.; Evans, M.; Harte, A.L.; Kumar, S. Lipopolysaccharide activates an innate immune system response in human adipose tissue in obesity and type 2 diabetes. Am. J. Physiol.-Endocrinol. Metab. 2007, 292, E740-E747. [CrossRef]

36. Peng, Z.; Chen, L.; Xiao, J.; Zhou, X.; Nüssler, A.K.; Liu, L.; Liu, J.; Yang, W. Review of mechanisms of deoxynivalenol-induced anorexia: The role of gut microbiota. J. Appl. Toxicol. 2017, 37, 1021-1029. [CrossRef] [PubMed] 
37. Ley, R.E.; Turnbaugh, P.J.; Klein, S.; Gordon, J.I. Microbial ecology: Human gut microbes associated with obesity. Nature 2006, 444, 1022-1023. [CrossRef] [PubMed]

38. Shin, N.R.; Whon, T.W.; Bae, J.W. Proteobacteria: Microbial signature of dysbiosis in gut microbiota. Trends Biotechnol. 2015, 33, 496-503. [CrossRef]

39. Wu, G.D.; Chen, J.; Hoffmann, C.; Bittinger, K.; Chen, Y.Y.; Keilbaugh, S.A.; Bewtra, M.; Knights, D.; Walters, W.A.; Knight, R.; et al. Linking long-term dietary patterns with gut microbial enterotypes. Science 2011, 334, 105-108. [CrossRef]

40. Liu, J.; Yue, S.; Yang, Z.; Feng, W.; Meng, X.; Wang, A.; Peng, C.; Wang, C.; Yan, D. Oral hydroxysafflor yellow A reduces obesity in mice by modulating the gut microbiota and serum metabolism. Pharmacol. Res. 2018, 134, 40-50. [CrossRef]

41. Garcia, J.P.; Adams, V.; Beingesser, J.; Hughes, M.L.; Poon, R.; Lyras, D.; Hill, A.; McClane, B.A.; Rood, J.I.; Uzal, F.A. Epsilon toxin is essential for the virulence of Clostridium perfringens type D infection in sheep, goats, and Mice. Infect. Immun. 2013, 81, 2405-2414. [CrossRef]

42. Shi, N.; Zhang, S.; Silverman, G.; Li, M.; Cai, J.; Niu, H. Protective effect of hydroxychloroquine on rheumatoid arthritis-associated atherosclerosis. Anim. Model. Exp. Med. 2019, 2, 98-106. [CrossRef]

43. Wang, B.; Kong, Q.; Li, X.; Zhao, J.; Zhang, H.; Chen, W.; Wang, G. A high-fat diet increases gut microbiota biodiversity and energy expenditure due to nutrient difference. Nutrients 2020, 12, 3197. [CrossRef] [PubMed]

44. Garber, A.; Hastie, P.; Murray, J.A. Factors influencing equine gut microbiota: Current knowledge. J. Equine Vet. Sci. 2020, 88, 102943. [CrossRef]

45. Tenorio-Jiménez, C.; Martínez-Ramírez, M.J.; Tercero-Lozano, M.; Arraiza-Irigoyen, C.; Del Castillo-Codes, I.; Olza, J.; Plaza-Díaz, J.; Fontana, L.; Migueles, J.H.; Olivares, M.; et al. Evaluation of the effect of Lactobacillus reuteri V3401 on biomarkers of inflammation, cardiovascular risk and liver steatosis in obese adults with metabolic syndrome: A randomized clinical trial (PROSIR). BMC Complement. Altern. Med. 2018, 18, 306. [CrossRef] [PubMed]

46. De Medina, F.S.; Romero-Calvo, I.; Mascaraque, C.; Martínez-Augustin, O. Intestinal inflammation and mucosal barrier function. Inflamm. Bowel Dis. 2014, 20, 2394-2404. [CrossRef]

47. Rivero-Gutiérrez, B.; Gámez-Belmonte, R.; Suárez, M.D.; Lavín, J.L.; Aransay, A.M.; Olivares, M.; Martínez-Augustin, O.; Sánchez de Medina, F.; Zarzuelo, A. A synbiotic composed of Lactobacillus fermentum CECT5716 and FOS prevents the development of fatty acid liver and glycemic alterations in rats fed a high fructose diet associated with changes in the microbiota. Mol. Nutr. Food Res. 2017, 61, 1600622. [CrossRef] [PubMed]

48. Carotti, S. Starring role of toll-like receptor-4 activation in the gut-liver axis. World J. Gastrointest. Pathophysiol. 2015, 6, 99-109. [CrossRef]

49. Weigert, C.; Hennige, A.M.; Lehmann, R.; Brodbeck, K.; Baumgartner, F.; Schäuble, M.; Häring, H.U.; Schleicher, E.D. Direct cross-talk of interleukin-6 and insulin signal transduction via insulin receptor substrate- 1 in skeletal muscle cells. J. Biol. Chem. 2006, 281, 7060-7067. [CrossRef]

50. Hotamisligil, G.S.; Peraldi, P.; Budavari, A.; Ellis, R.; White, M.F.; Spiegelman, B.M. IRS-1-mediated inhibition of insulin receptor tyrosine kinase activity in TNF- $\alpha$ - and obesity-induced insulin resistance. Science 1996, 271, 665-668. [CrossRef]

51. Tse, M.C.L.; Herlea-Pana, O.; Brobst, D.; Yang, X.; Wood, J.; Hu, X.; Liu, Z.; Lee, C.W.; Zaw, A.M.; Chow, B.K.C.; et al. Tumor necrosis factor- $\alpha$ promotes phosphoinositide 3-kinase enhancer A and AMP-activated protein kinase interaction to suppress lipid oxidation in skeletal muscle. Diabetes 2017, 66, 1858-1870. [CrossRef] [PubMed]

52. Peña, J.A.; Versalovic, J. Lactobacillus rhamnosus GG decreases TNF- $\alpha$ production in lipopolysaccharide-activated murine macrophages by a contact-independent mechanism. Cell. Microbiol. 2003, 5, 277-285. [CrossRef] [PubMed]

53. Calcinaro, F.; Dionisi, S.; Marinaro, M.; Candeloro, P.; Bonato, V.; Marzotti, S.; Corneli, R.B.; Ferretti, E.; Gulino, A.; Grasso, F.; et al. Oral probiotic administration induces interleukin-10 production and prevents spontaneous autoimmune diabetes in the non-obese diabetic mouse. Diabetologia 2005, 48, 1565-1575. [CrossRef] [PubMed]

54. Furet, J.P.; Kong, L.C.; Tap, J.; Poitou, C.; Basdevant, A.; Bouillot, J.L.; Mariat, D.; Corthier, G.; Doré, J.; Henegar, C.; et al. Differential adaptation of human gut microbiota to bariatric surgery-induced weight loss: Links with metabolic and low-grade inflammation markers. Diabetes 2010, 59, 3049-3057. [CrossRef] 